\title{
Medievalista
}

Online

$30 \mid 2021$

Número 30

\section{Fonti e contesti dei miracoli eucaristici delle Cantigas de Santa María 128 e 208}

Sources and context of the eucharistic miracles of the Cantigas de Santa María 128 and 208

\section{Manuel Negri}

\section{OpenEdition}

\section{Journals}

Edizione digitale

URL: https://journals.openedition.org/medievalista/4548

ISSN: 1646-740X

\section{Editore}

Instituto de Estudos Medievais - FCSH-UNL

Notizia bibliografica digitale

Manuel Negri, «Fonti e contesti dei miracoli eucaristici delle Cantigas de Santa María 128 e 208» Medievalista [Online], 30 | 2021, posto online no dia 01 julho 2021, consultado o 24 julho 2021. URL http://journals.openedition.org/medievalista/4548

Questo documento è stato generato automaticamente il 24 juillet 2021

\section{(c) (†) 8}

Mediavalista está licenciado com uma Licença Creative Commons - Atribuição-NãoComercial 4.0 Internacional. 


\section{Fonti e contesti dei miracoli eucaristici delle Cantigas de Santa María 128 e 208}

Sources and context of the eucharistic miracles of the Cantigas de Santa María 128 and 208

Manuel Negri

\section{NOTA DELL'EDITORE}

Data recepção do artigo / Received for publication: 3 de Julho de 2020

Data aceitação do artigo / Accepted in revised form: 22 de Abril de 2021

1 Le Cantigas de Santa María - canzoni dedicate alla Vergine Maria nella seconda metà del XIII secolo ${ }^{1}$ - sono il risultato della confluenza alla corte del suo patrocinatore Alfonso $\mathrm{X}$ di Castiglia (nonché probabile autore, per alcune di esse), di varie tradizioni miracolistiche, di natura e provenienza diversa.

2 Semplificando, se per alcuni testi - soprattutto quelli che formano la prima parte della collezione mariana - il modello dei miracoli pare derivare dalle grandi e note collezioni di prodigi della Vergine già ampiamente conosciuti in tutto lo spazio europeo, per altri - molto più complessi da analizzare - si ipotizza invece l'apporto di tradizioni più legate alla cultura popolare, locale (miracoli connessi a qualche santuario), o anche provenienti dalle Vitae di altri santi della cultura occidentale, opportunamente riadattate al nuovo contesto mariano ${ }^{2}$.

Quali siano stati comunque i modelli di riferimento per Alfonso X e il suo entourage di collaboratori, essi non si mostrano mai univoci nella formazione di ogni CSM: per questo è sempre preferibile parlare non tanto di una sola fonte, ma di più varianti o tradizioni rispetto ad un medesimo miracolo (o parti di esso) che il laboratorio mariano del Re Sapiente ha saputo rimaneggiare all'insegna di una ricercata originalità. 
Questo fatto determina dunque la difficoltà nel tentare di rintracciare la fonte $(0$, più propriamente, le fonti) delle CSM che formano l'intero corpus mariano; e, tra i testi 'difficili' da analizzare in questo senso, in virtù delle dinamiche non lineari nel processo di trasmissione di una tradizione, si trovano anche quelli oggetto di questo breve studio.

5 Si tratta di quelle CSM che trasmettono dei miracoli dove i sacri azzimi si trasformano in carne e sangue per ribadire la presenza reale di Cristo in quelle sostanze e, eventualmente, impedire che le ostie vengano utilizzate in rituali profani. In altri prodigi, quest'ultime si trasfigurano in una immagine della Vergine o di Gesù, sempre in risposta ad un atto di profanazione o ad una funzione religiosa celebrata con scetticismo nei confronti del sacramento dell'Eucaristia ${ }^{3}$.

6 In alcuni casi, questi prodigi possono anche coesistere nello stesso miracolo, ma sempre con le medesime finalità: confermare il dogma della transustanziazione ed impedire un utilizzo di quel sacramento in rituali che si potrebbero definire 'magici'. In totale, son ben quattro i componimenti che possono essere ricondotti a queste tematiche: CSM $104,128,149$ e $208^{4}$.

7 Come già accennato, l'obiettivo di questo studio è quello di presentare delle nuove ipotesi sui precedenti narrativi di due delle CSM appartenenti a questo gruppo, le CSM 128 e 208. La scelta di restringere il campo obbedisce a due criteri: il primo, ovviamente, è quello dello spazio offerto da questa pubblicazione; il secondo invece ha a che vedere con la natura degli stessi miracoli: si tratta infatti di testi che condividono una situazione apparentemente simile dove un'ostia consacrata, dopo essere stata inserita all'interno di un alveare, inizia ad essere adorata da alcune api, divenendo infine oggetto di una vistosa trasfigurazione ${ }^{5}$.

\section{La CSM 128}

9 Nella CSM 128 si racconta la storia di un apicoltore delle Fiandre che, per incrementare la produzione di miele e cera, ha bisogno di più api. Vuole però sforzarsi il minimo possibile. A tal proposito, un'anziana fattucchiera del posto gli consiglia di ricevere l'ostia durante la messa per poi conservarla tra la lingua e il palato e riporla, una volta fatto ritorno a casa, in una delle sue arnie. Arriva il giorno di raccolta del miele, ed ecco che, riaperta l'arnia, si trova davanti ad un fatto straordinario: i sacri azzimi hanno preso le sembianze della Vergine Maria con, in braccio, il piccolo Gesù (vv32-36)

"E abriu aquela logo, u a osti' ensserrara,

e viu [y] Santa Maria, mui fremosa e mui crara,

con seu Fillo Jhesu-Cristo en seus braços, que criara,

que tĩia abraçado e el ela abraçada.

Tan muit' é con Jesu-Cristo Santa Maria juntada ...".

Turbato da questa rivelazione, il peccatore corre ad informare il prete del luogo. Quest'ultimo non tarda poi molto a recarsi sul posto per comprovare coi propri occhi il miracolo. Ordina allora di riunire i fedeli affinché tutti abbiano la possibilità di contemplare la sacra rappresentazione, in preghiera. Nel frattempo, già all'arrivo dei primi parrocchiani, un profumo dolcissimo - "migliore di quello dei gigli, delle viole o dell'acqua di rose" - inizia a diffondersi tutt'intorno, quasi a voler accompagnare lo straordinario evento (vv52-56):

“Logo foron alá todos e viron en com' estava

na colmẽa a mui santa Virgen e com' abraçava

a seu Fillo Jhesu-Cristo, e mui mellor odor dava 
que liros nen violetas non dan, nen agua rosada.

Tan muit' é con Jesu-Cristo Santa Maria juntada ..."

11 L'arnia è infine portata in processione per le strade della cittadina fino alla chiesa, tra la gente festosa e piena di devozione. Qui viene adagiata sopra l'altare maggiore per poter essere vegliata fino a tarda notte dai fedeli che erano rimasti a pregare. Ed è proprio durante la veglia che si verifica l'ultimo prodigio: i sacri azzimi ritornano pian piano ad assumere il loro aspetto originario.

\section{Motivi e precedenti}

13 Alcuni frammenti di questa situazione miracolistica si possono rintracciare sia nel repertorio curato da Stith Thompson che in quello di Frederich Tubach, rispettivamente nei motivi B259.4 ("Bees build church of wax to contain consecrated host") e 2662 ("Host in bee hive. A woman hides the Host in a hollow tree. She forgets it for a year and then finds bees have built a shrine of honeycombs around it") ${ }^{7}$.

14 È da notare però che le situazioni registrate si rifanno solamente a due delle possibili varianti di questa tipologia di miracolo eucaristico: non sempre, infatti, le api si impegnano a costruire una struttura a protezione dell'ostia, limitandosi a volte a mantenere unicamente incorrotta la sacra forma in vista di una sua eventuale trasfigurazione. Quest'ultimo è proprio il caso che offre la CSM 128, la quale - in virtù di questa soluzione narrativa - sembra rifarsi precisamente a tre dei precedenti miracolistici tra quelli censiti in occasione di questo studio. Le versioni interessate vengono evidenziate in grigio nella tabella seguente ${ }^{8}$ :

\begin{tabular}{|l|l|l|l|l|l|}
\hline $\mathbf{N}^{\circ}$ & Autore & Coll./Ms. & Carte & Capitolo & Periodo \\
\hline $\mathbf{1}$ & Pietro di Cluny & De Miraculis & $/$ & I, 1 & Inizi XII sec. \\
\hline $\mathbf{2}$ & Erberto di Chiar. & De Miraculis & $/$ & III, 30 & 1178 \\
\hline $\mathbf{3}$ & Giraldo Cambrense & Gemma Eccl. & $/$ & I, 11 & 1199 \\
\hline $\mathbf{4}$ & Anon. & Rivipullensis 193 & $44 \mathrm{v}-45 \mathrm{~V}$ & 15 & Fine XII sec. \\
\hline $\mathbf{5}$ & Anon. & Par. lat. 14958 & $161 \mathrm{rv}$ & $/$ & XIII sec. \\
\hline $\mathbf{6}$ & Cesario di Heisterbach & Dial. Miraculorum & & IX, 8 & Inizio XIII sec. \\
\hline $\mathbf{7}$ & Jacques de Vitry & Serm. Vulgares & $/$ & 191 & Inizio XIII sec. \\
\hline $\mathbf{8}$ & Anon. & Liber exemplorum & $/$ & 16 & XIII sec. \\
\hline $\mathbf{9}$ & Stefano di Borbone & Tractatus & $/$ & 317 & XIII sec. \\
\hline $\mathbf{1 0}$ & Tommaso di Cantimpré & Bonum universale & $/$ & II, 40,1 & $1256-1263$ \\
\hline $\mathbf{1 1}$ & Anon. & Royal 7 D. i. & $66 \mathrm{v}$ & 27 & Fine XIII sec. \\
\hline
\end{tabular}

15 La prima versione che deve essere confrontata col testo della CSM 128 è quella trasmessa dall'opera De Miraculis di Pietro il Venerabile, risalente al termine del sec. XII. In apertura del primo capitolo, Pietro racconta che un rusticus originario dell'Alvernia 
possedeva alcuni alveari. Temendo che la produzione delle sue arnie potesse andare incontro a dei problemi, un giorno decise di premunirsi contro tali avversità servendosi della magia. Gli venne consigliato infatti di recarsi in chiesa, prendere la comunione e conservare intatti i sacri azzimi in bocca per poi, una volta ritornato a casa, scegliere una delle sue arnie per soffiarvi all'interno con questi ancora tra la lingua e il palato":

"Erat in Arvernico territorio rusticus quidam habens alvearia, in quibus examina apum melleam favorum dulcedinem conficere solent. Quas jam dictus rusticus aut evolare, aut emori, aut aliquo casu sibi deperire pertimescens usus pravo sortilegorum consilio, qui diabolica operatione, etiam de beneficiis Dei maleficia facere consueverunt, et (quod est dictu intolerabile) ipsis quoque divinis sacramentis per artes magicas abutuntur, accessit ad ecclesiam, et sumpto a sacerdote (ut mos est Christianorum) corpore Dominico, illudque ore retinens, nec glutire volens, ut jam doctus fuerat, ad unum de jam dictis alvearibus, in quo apes continebantur, accessit, atque foramini, quod in eo erat ore adaptato, sufflare coepit".

Questo avrebbe inoltre garantito - come riferito allo stesso rusticus - non solo la protezione ai suoi operosi insetti, ma anche una maggiore produzione di miele e di cera. Mise dunque in atto il rituale soffiando quanto più poteva, ma proprio a causa della forza esercitata nell'atto, oltre all'aria espulse dalla bocca anche la stessa ostia, che cadde così a terra. A quel punto, in sua presenza, accadde un primo fatto straordinario: le api, come esseri dotati improvvisamente di razionalità e di pia devozione, accorsero in soccorso dei sacri azzimi e li trasportarono all'interno dell'alveare:

"Nam dictum sibi fuerat quod si corpore Domini ore retento apibus in vase constitutis insufflaret, nulla deinceps moreretur, nulla recederet, nulla deperiret; sed omnibus integre conservatis, de augmento fructus multo amplius quam ante gauderet [...] Cumque avidus lucri anhelitum protrahens vehementer sufflaret, ipso linguae et aeris impulsu jactum Dominicum corpus juxta vas illud ad terram corruit. Cum ecce omnis illa apum multitudo de intimis egressa, ad corpus Domini sui reverenter accurrit, atque in morem rationabilium creaturarum de terra sublevatum, habitaculis suis cum multa veneratione, homine illo conspiciente, induxit".

17 In un primo momento, volendo forse non credere a quello che aveva visto, l'uomo decise di allontanarsi per occuparsi di altre faccende. In seguito, iniziò però a temere di aver fatto male lasciando che l'ostia rimanesse all'interno dell'arnia. Quindi tornò sui propri passi e, pur di recuperare il Corpo di Cristo, si mostrò disposto ad eliminare quelle stesse api che aveva cercato poco prima di proteggere con un incantesimo. E così fece, gettandovi sopra dell'acqua. Una volta morte, le api rivelarono nella parte più recondita dell'alveare l'ostia che era uscita dalla sua bocca, intatta, quasi come l'avessero voluta proteggere; ma, con grande sorpresa, si poté anche constatare come il pane azzimo avesse assunto le sembianze di Gesù bambino - ancora in fasce circondato dal miele ${ }^{10}$ :

"Quibus exstinctis, dum intima favorum, quos sibi recondere et conservare volebat, rimaretur, ecce, (mirabile dictu!) conspicit corpus Dominicum, quod ex ejus ore lapsum fuerat, in formam speciosissimi pueri, veluti cum recens nascitur, immutatum, inter favos et mella jacere". sacra rappresentazione all'interno di un alveare - sembrerebbe poi essere confluito in due collezioni miracolistiche elaborate tra la fine del XII e gli inizi del XIII sec., 
suggerendo così una circolazione del prodigio anche in un periodo più prossimo all'attività poetica di Alfonso $\mathrm{X}$.

Lo si ritrova infatti dapprima all'interno di una collezione di exempla moralia - che si estende tra i fols. 7v-156v e poi, in modo disordinato, fino al fol. 161rv - trasmessa dal miscellaneo Paris, Bibliothèque Nationale de France, Ms. Par. lat. 14958, proveniente dallo scriptorium di San Vittore ${ }^{11}$. Il miraculum, rubricato "De rustico qui posuit corpus domini cum apibus ut augmentaretur", non è altro che un rifacimento del prodigio raccontato da Pietro il Venerabile, dal quale si riprendono anche interi sintagmi opportunamente adattati ad uno stile fortemente ipotattico ${ }^{12}$ :

[fol. 161v] "Legitur esse in avernie territorio quemdam rusticum . examen apum habundantem an alveariis . quas ipse pertimens perdere . aut mori . sive alis aliquo casu deperire . usus tamen pravo consilio scilicet sortilegum dyabolica operatione . qui de beneficiis dei maleficia facere consueverunt . qui etiam divinis sacramentis abutuntur . supradictus rusticus ad ecclesiam communicaturus accessit . et sumpto a sacerdote corpore dominico ut christianis mos est . illud in ore suo retinens . glutire noluit . set clauso ore illud ad unum de alveariis suis portavit . atque foramini quod in eo erat ore adaptato . ut edoctus fuerat . sufflare cepit . tamquam illa sufflatione $(. . .)^{13}$ deinceps esse moritura . nulla recessura . nulla peritura . nulla steriler futura sed omnis ibi semper remanerent . et fructum habundantissime facerent . Cum igitur flatum ad interiora toto conatu emitteret . avidus enim luc $<$ ri $>14$ hanelitu $m$ protrahebat vehementis insufflans . ipso lingue impulsu iactatum meante . corpus dominicum . iuxta illud vas ad terram corruit . et ecce omnes apes ille reverenter accurrunt . et tamquam creature rationales . corpus domini de terra sublevatum habitaculis suis . rustico inspectante introducuunt. quod negligens ac parvipendens . ad sua negotia se convertit . Sed cum iter ag<eret> repentino et intolerabili timore turbatus . se nequiter egisse (...) compunctus retro pedem tulit . atque in vindictam sceleris sui . apes quarum vitam opere nefario conservare voluerat . superiecta multa aque violentia enecavit . quibus extinctis . dum favorum intima rimaretur et . corpus dominicum in forma spetiossissima infantis recenter nati conspicit . sed mortui . inter favos et mella iacere. Hesitans autem quod inde faceret . tandem cogitavit eum in manibus accipere et ad ecclesiam referre . et ibi nemine sciente tamquam infantem mortuum tumulare. Quod dum ita deferret . subito ab indignis manibus ferentibus . invisibiliter elapsus disparuit“".

Una seconda attestazione del prodigio, in una veste già 'marianizzata' - dove cioè è espressamente la Vergine Maria ad intercedere affinché possa realizzarsi il miracolo della trasfigurazione dell'ostia $-{ }^{15}$, la si trova all'interno di una collezione anonima di miracula virginis trasmessa ai nostri giorni da due mss., il più antico dei quali è databile tra la fine del XII e gli inizi del XIII sec. ${ }^{16}$. Si tratta di una raccolta che solamente a partire dalla metà del secolo scorso è stata rimessa sotto i riflettori dalla critica e ricondotta - per alcuni prodigi - anche ad altri récits presenti nella collezione mariana del Re Sapiente. Nel manoscritto più antico che la trasmette (Barcelona, Archivo General de la Corona de Aragón, Ms. Rivipullensis 193, fols. 27v-48r ${ }^{17}$, proveniente dal monastero catalano di Ripoll, tra i fols. $44 \mathrm{v}$ e $45 \mathrm{v}$ si può leggere infatti quanto segue ${ }^{18}$ :

“Tempore Karoli magni imperatoris, in partibus Flandrie, terricola quidam, instigante sibi | quedam [fol. 45r] phitonissa, ut apes vicinorum ad suum venirent apiarium, et mellis copiam magis pre ceteris haberet vicinis, eucaristiam paschalem, quod in ecclesia beate Marie virginis a sacerdote acceperat, in quodam vase apium misit ${ }^{19}$. Cum vero tempus colligendi mel adveniret, et ipse vas disco cooperiret, pro melle factorem mellis invenit. Quid plura? Res mira, ante et post nec audita. Divam Virginem inter ulnas sacras puerum suum pium Ihesum tenentem in vase, ubi eucharistia immiserat, reperit stantem. Quod videns miser ille valde obstupuit. Tunc exivit tanta claritas et bonus odor a vase, quod totus locus ille 
mirabiliter refulsit. Idcirco stultus ille amplius ibi stare ${ }^{20}$ nequivit, sed ecclesie sacerdotem adiit, et rem gestam per ordinem ei narravit. Sacerdos vero ${ }^{21}$, ut sapiens, rem prius per nuncios probata, pulsatis prolixe tintinabulis, coadunata maxima multitudine parrochianorum suorum, indutus sacerdotalibus vestimentis, cum clericis, et cruce, et turibulis ivit psalmocinando ad locum $\mathrm{ubi}^{22}$ vas erat, rustico duce. Cum autem tanta dei mirabilia intuitus esset, flexis genibus cum magnis singultibus et lacrimis, accepit vas inter brachia sua ${ }^{23}$, ad ecclesiam tulit. Tunc cecinit diligenter matutinas paschales et horas, primam scilicet et terciam, sicuti egerat die qua tradiderat eucharistiam rustico stulto. Tunc posuit divam Virginem cum puero amicabiliter | super altare, et cecinit cum illa vera hostia sola missam [fol. 45v] paschalem. Cum autem diceret oracionem que est post pacem dominicam, ubi debet hostia frangi, ecce puer oblatus, hostia illa vera, vertitur in hostia panis, illa videlicet hostia, quam rustico dederat die paschali". autonoma nel laboratorio mariano del Re Sapiente a partire da un comune precedente agiografico qualsiasi, quello che segue contribuisce a rafforzare i sospetti sulla conoscenza da parte di Alfonso della 'tradizione' rappresentata dalla versione di Ripoll. È da notare infatti come in entrambi i récit si insista su alcuni fenomeni che accompagnano il prodigio, come quello olfattivo, condiviso in esclusiva dal testo alfonsino e da quello di provenienza catalana: un profumo meraviglioso che si diffonde per tutto l'ambiente circostante a partire proprio dall'alveare che contiene la sacra forma mutata d'aspetto ${ }^{24}$.

L'ipotesi che la chiave di volta capace di risolvere l'enigma di una delle fonti principali (non l'unica, ovviamente) consultate da Alfonso $\mathrm{X}$ e dal suo entourage per la composizione della CSM 128 possa essere la versione trasmessa dal manoscritto di provenienza catalana ${ }^{25}$ - e forse comune a quella seguita dai collaboratori del Re Sapiente a partire da un condiviso antecedente di provenienza francese - potrebbe rafforzarsi nel notare come i debiti alfonsini con questa tradizione siano stati già evidenziati in relazione almeno ad altre due CSM che occupano una posizione relativamente prossima a quella oggetto di questo studio. Tale vicinanza suggerirebbe pertanto un rilievo miracolistico effettuato in primis a partire dalla stessa collezione, 
disponibile tra le mani dei collaboratori alfonsini in una fase puntuale del lavoro. Si tratta inoltre di miracoli poco o per nulla trasmessi dalla rimanente tradizione miracolistica a noi nota.

Il primo caso riguarda la CSM 138, dove si narra un miracoloso intervento di guarigione da parte della Vergine Maria a beneficio del santo patriarca Giovanni Crisostomo il cui miracolo parrebbe costituire l'esito di un processo di 'marianizzazione' di una vicenda narrata per la prima volta nel Dialogus de Vita S. Joannis Chrysostomi scritto da Palladius tra il 408 e il $412 \mathrm{ca}$. Si tratta infatti di un prodigio già legato alla figura mariana proprio nella collezione di Ripoll e di cui non si hanno notizie sulla sua trasmissione al di fuori di quest'ultima (dove occupa la terza posizione).

Il secondo caso è quello della CSM 141, la quale mette in scena la ben nota storia del monaco inglese ringiovanito da Maria. Anche per questo prodigio si conosce una diffusione assai limitata nel panorama miracolistico europeo e uno dei precedenti del testo alfonsino si può ritrovare esclusivamente nella collezione tràdita per la prima volta dal ms. di Ripoll (dove occupa la settima posizione) ${ }^{26}$. trascurabili. Il protagonista stavolta è un eretico di Tolosa che continua a partecipare alla messa per dissimulare agli occhi della comunità dei fedeli in Cristo di cui fa parte la propria miscredenza. dubbi sui sacri azzimi -, decide di conservarli in bocca fino al termine della messa con già, in mente, un disegno ben preciso ${ }^{27}$. Una volta tornato a casa, li mette dentro un'arnia forse per vedere se le api inizino a costruirvi (obrar) qualcosa attorno, o anche solo per verificarne il comportamento in presenza dell'ostia (vv30-34) ${ }^{28}$ :

"E en hũa ssa colmẽa o deitou e diss' assi:

"Abellas, comed' aquesto, ca eu o vinno bevi;

e se vos obrar sabedes, verei que faredes y".

E des i foi-sse mui ledo o traedor descreudo.

Aquele que ena Virgen carne por seer veudo ... ".

Arrivato il giorno della raccolta del miele e della cera, l'eretico apre l'arnia e vi trova all'interno una piccola cappella munita di altare; e sopra a quest'ultimo, una rappresentazione in miniatura della Vergine Maria che stringe fra le sue braccia il piccolo Gesù. La scoperta di questo fatto prodigioso viene inoltre accompagnata da un profumo magnifico, capace di convertirlo all'istante (vv40-44):

“Abriu mui tost' a colmẽa e hũa capela viu

con seu altar estar dentro, e a omagen cousiu

da Virgen cono seu Fillo sobr' ele, e ar sentiu

un odor tan saboroso que logo foi convertudo,

Aquele que ena Virgen carne por seer veudo..."

Dopo questo fatto, l'uomo decide di andare dal vescovo per confessarsi. Come nel miracolo precedente, anche qui il religioso organizza in seguito una grande processione per esaminare di persona i sacri azzimi e per riunire i fedeli in preghiera.

\section{Motivi e precedenti}

34

Come già anticipato, una delle differenze principali rispetto al récit della CSM 128 consiste nel fatto che, oltre alla trasfigurazione dell'ostia, si assiste qui anche alla costruzione di una struttura votiva a sua protezione. 
La presenza di questo specifico motivo 'di peso' (o variante) porta ora a selezionare altre occorrenze tra quelle già presentate nella tabella precedente da mettere a confronto con la CSM 208 (vengono sempre evidenziate in grigio):

\begin{tabular}{|l|l|l|l|l|l|}
\hline $\mathbf{N}$ & Autore & Coll./Ms. & Carte & Capitolo & Periodo \\
\hline $\mathbf{1}$ & Pietro di Cluny & De Miraculis & $/$ & I, 1 & Inizi XII sec. \\
\hline $\mathbf{2}$ & Erberto di Chiar. & De Miraculis & $/$ & III, 30 & 1178 \\
\hline $\mathbf{3}$ & Giraldo Cambrense & Gemma Eccl. & $/$ & I, 11 & 1199 \\
\hline $\mathbf{4}$ & Anon. & Rivipullensis 193 & $44 \mathrm{v}-45 \mathrm{~V}$ & 15 & Fine XII sec. \\
\hline $\mathbf{5}$ & Anon. & Par. lat. 14958 & $161 \mathrm{rv}$ & $/$ & XIII sec. \\
\hline $\mathbf{6}$ & Cesario di Heisterbach & Dial. Miraculorum & & IX, 8 & Inizio XIII sec. \\
\hline $\mathbf{7}$ & Jacques de Vitry & Serm. Vulgares & $/$ & 191 & Inizio XIII sec. \\
\hline $\mathbf{8}$ & Anon. & Liber exemplorum & $/$ & 16 & XIII sec. \\
\hline $\mathbf{9}$ & Stefano di Borbone & Tractatus & $/$ & 317 & XIII sec. \\
\hline $\mathbf{1 0}$ & Tommaso di Cantimpré & Bonum universale & $/$ & II, 40,1 & $1256-1263$ \\
\hline $\mathbf{1 1}$ & Anon. & Royal 7 D. i. & $66 \mathrm{v}$ & 27 & Fine XIII sec. \\
\hline
\end{tabular}

Il primo autore noto a dar conto del motivo della costruzione di un santuario di miele e di cera a protezione dell'ostia in circostanze simili a quelle della CSM 208 - in un exemplum pienamente eucaristico - è Giraldo Cambrense nella sua Gemma Ecclesiastica compilata attorno al 1199.

Tuttavia, nell'undicesimo capitolo della Distinctio I, l'autore racconta che l'ostia non era stata inserita all'interno di un'arnia, bensì in una cavità nel tronco di un albero da parte di un uomo che nutriva dei dubbi nei confronti della sacra forma e delle sue capacità (altra variante significativa di questa tipologia di exempla) ${ }^{29}$. Si intende comunque che la motivazione di questo gesto ubbidiva sempre al desiderio - alimentato da certe credenze - di poter avere a disposizione una notevole produzione di miele e di cera senza particolare sforzo, sfruttando proprio il potere che si credeva insito nell'ostia consacrata (fatto, del resto, che accomuna quasi tutti i prodigi censiti fino a questo punto).

Un prodigio che, oltre ad imperniarsi su questo stesso motivo, mostra anche più affinità nelle modalità di realizzazione dello stesso, ricompare invece nel corso delle prime due decadi del secolo successivo nel Dialogus Miraculorum di Cesario di Heisterbach.

Qui, nell'ottavo miracolo del IX capitolo dell'opera (De apibus quae basilicam corpori Dominico fabricaverunt), si racconta infatti che una donna si era trovata in difficoltà per aver constatato che le sue api non fossero produttive a sufficienza. Temendo per il loro stato di salute e alla ricerca di qualche rimedio, le venne detto - come sempre - di collocare in mezzo a loro un'ostia consacrata. Si recò dunque in chiesa, prese la 
comunione, nascose i sacri azzimi in bocca, e, una volta ritornata a casa, li collocò all'interno di uno dei suoi alveari.

In seguito, anche stavolta, il prodigio non si fece attendere: le api cominciarono subito a costruire attorno all'ostia una piccola cappella per poi adagiarla sopra un altare minuto. Il miracolo venne infine comunicato dalla donna al sacerdote dal quale aveva ricevuto la comunione, mostrandosi pentita per quanto aveva fatto ${ }^{30}$ :

"Vermiculi Creatorem agnoscentes, de favis suis dulcissimis hospiti dulcissimo capellulam mirae structurae fecerunt, in qua altare eiusdem materiae erigentes, sacratissimum corpus super illud posuerunt [...] Processu temporis cum femina idem vasculum aperuisset, et iam dictum oratorium considerasset, expavit, currensque ad sacerdotem, confessa est ei omnia et quae egit et quae vidit".

Il prete, raccolti alcuni parrocchiani, si recò quindi dove si trovava l'ostia e così potè notare le api che le volavano attorno rivolgendo lodi al Creatore. Una volta diradati gli insetti, tutti poterono infine ammirare quello che era stato costruito attorno ai sacri azzimi e di cui Cesario offre una descrizione particolareggiata: una cappella con finestre alle pareti, campanile, tetto, porta ed altare ${ }^{31}$ :

"Tunc ille assumptis secum parochianis suis ad vasculum venerunt, apes circumvolantes et in laudem Creatoris bombizantes abegerunt, capellulae parietes, fenestras, tectum, campanile, ostium, et altare admirantes, corpus Domini cum laude et gloria ad ecclesiam retulerunt."

Se nei Sermones Vulgares di Giacomo da Vitriaco e nell'anonimo Liber Exemplorum di ambiente francescano (entrambi coevi al periodo di compilazione del Dialogus di Cesario) era stato ripreso il récit in una veste più affine a quella trasmessa da Giraldo Cambrense - dunque con la variante dell'ostia che, invece di essere inserita all'interno di un'arnia, era stata nascosta in una cavità arborea $-{ }^{32}$, quello che racconta Stefano di Borbone attorno alla metà del XIII sec. riprende invece quanto narrato da Cesario.

Di nuovo, infatti, viene presentata la vicenda di un rusticus che desiderava moltiplicare le sue api $\mathrm{e}$, in tal modo, ottenere più miele e cera. $\mathrm{E}$, anche qui, si ripete lo stesso miracolo, con le api che avevano cominciato a costruire attorno al Corpo di Cristo una piccola chiesa, completa di fondamenta, colonne ed altare (anche in questo caso, la descrizione della struttura si rivela particolarmente dettagliata) ${ }^{33}$ :

"Tunc omnes apes ad alveoloum ubi erat corpus Christi convenerunt, et, pro irreverencia sibi facta compacientes quasi, opere suo inceperunt construere ecclesiam parvulam, fundamenta et bases et columpnas erigere, et altare consimili opere, et corpus dominicum cum maxima reverencia super illud altare collocaverunt, et ecclesiolam illam miro opere et pulcherrimo perfecerunt infra alveolum illum".

Poco tempo dopo, come di consueto, il miracolo venne comprovato dall'autorità ecclesiastica del luogo, dopo che il rusticus non era più riuscito ad avvicinarsi alle arnie a causa di un attacco subito dalle stesse api; e così anche gli occhi del sacerdote poterono verificare la presenza di una parvulam ecclesiam e di un altare sul quale era stato collocato il Corpo di Cristo. 
prossimità culturale e politica; motivazioni che, del resto, lo studioso aveva già avuto modo di esporre nei suoi lavori ${ }^{34}$.

Questa ipotesi sembrerebbe legittima anche da un punto di vista cronologico escludendo Stefano di Borbone, autore troppo contemporaneo al periodo di attività poetica del Re Sapiente - e tenendo conto dell'assenza del motivo in altre collezioni immediatamente precedenti: il solo autore infatti che avrebbe potuto fornire al sovrano castigliano-leonese il motivo (o il suggerimento) della costruzione di una struttura votiva a protezione dell'ostia potrebbe essere stato solamente Cesario, già frequentato del resto per altri testi.

In questo caso, però, a differenza di quanto si poteva concludere per la CSM 128, la questione si mostra meno lineare. Non si deve infatti dimenticare che - anche ipotizzando come fonte principale il récit di Cesario per la tematica più importante della narrazione - persistono delle differenze sostanziali tra questo e la CSM 208; ma si può anche verificare come queste divergenze, in realtà, non siano poi così discriminanti, in quanto potrebbero anche essersi generate per via di un nuovo contesto sociale e dottrinale in cui si trovava immerso lo scriptorium alfonsino.

In questo senso, dunque, il risultato finale potrebbe anche essere la conseguenza di un aggiornamento dell'exemplum tradizionale in base a certi parametri, e non necessariamente il risultato della consultazione di qualche fonte a noi ignota ${ }^{35}$.

Si consideri, ad esempio, il primo elemento di difformità tra la CSM 208 e il racconto del Dialogus: la tipologia del protagonista. Se, infatti, nel récit di Cesario si è in presenza di una donna che sembra allevare delle api per mestiere e che agisce nell'ottica di avere un maggiore tornaconto economico, nella CSM troviamo un eretico che, più che essere mosso dal desiderio di incrementare la produzione di miele e cera delle sue arnie, finisce col profanare l'ostia per 'metterla alla prova' e quindi per verificare che, con la sua presenza all'interno dell'arnia, anche una parte del mondo animale potesse piegarsi alla volontà divina, come guidata da una intelligenza superiore.

In una versione apparsa molto più tardi di questo prodigio, ovvero agli inizi del XIV sec., in una collezione anonima di miracoli oggi trasmessa dal London, British Library, Ms. Additional 27, 336 e riconducibile con buona probabilità ad un ambiente francescano dell'Italia settentrionale, il protagonista di questa storia è ancora un eretico che, dopo essere stato beneficiato da un miracolo simile, alla fine si pente e si converte $^{36}$, proprio come accade nella CSM 208.

Si potrebbe trattare di una testimonianza seriore di un récit magari precedente all'attività poetica del saggio re castigliano-leonese? Non necessariamente: è più probabile che, su questo punto, anche Alfonso $\mathrm{X}$ avesse voluto riadattare questo prodigio rispondendo così alle necessità manifestate dalla Chiesa soprattutto negli anni successivi al IV Concilio Lateranense che aveva fissato come uno dei punti cardine del programma la lotta alle eresie; e che abbia voluto fare di questo nuovo exemplum mariano una sorta di manifesto per ribadire non solo la necessità di mettere alla berlina gli eretici, ma anche di ricordare la sacralità del Corpo di Cristo, posta in dubbio a quel tempo da parte di molti, ma soprattutto dalla corrente catara ${ }^{37}$.

La seconda differenza è che nella CSM 208 accade qualcosa di significativo che non trova riscontro in nessun'altra versione anteriore: l'ostia che viene posata sul piccolo altare costruito dalle api subisce anche una trasfigurazione in una rappresentazione 
mariana. Nella tradizione rimanente, al contrario, non vi è nessuna trasformazione apparente quando compare il motivo della costruzione della cappella cerea ${ }^{38}$.

Di fronte a questo unicum nella tradizione, è economico ipotizzare che su questo punto Alfonso abbia voluto operare una fusione tra due motivi, guidato dalla necessità di fornire, soprattutto in un miracolo tradizionalmente cristologico come quello delle ostie soggette a trasfigurazione, un segnale della presenza mariana negli stessi azzimi, ribadendo così il ruolo di Maria come figura capace di intercedere anche per questa tipologia di miracoli. innovazioni volte non solo a rispondere alle necessità dottrinali del suo tempo, ma anche a venire incontro ai gusti dell'epoca. Ne sarebbero prova, come già precisato, il cambiamento di protagonista (non solo di genere) e una vera e propria fusione di due motivi miracolistici per dare più centralità alla figura di Maria, vera ed unica protagonista del suo canzoniere.

\section{BIBLIOGRAFIA}

Fonti manoscritte

Barcelona, Archivo General de la Corona de Aragón, Ms. Rivipullensis 193. 
Paris, Bibliothèque Nationale de France, Ms. Par. lat. 14958.

Fonti scritte

ALFONSO X, El Sabio - Cantigas de Santa María. Cantigas 101 a 260. II. Ed. Walter Mettmann. Madrid: Castalia, 1988.

CAESARIUS HEISTERBACENSIS - Dialogus Miraculorum. Vol. I. Ed. Joseph Strange. Coloniae: Bonnae et Bruxellis, Sumptibus J. M. Heberle (H. Lempertz \& Comp.), 1851.

ÉTIENNE DE BOURBON - Tractatus de diversis materiis praedicabilibus. Ed. Albert Lecoy de la Marche. Paris: Librairie Renouard, 1877.

GIRALDUS CAMBRENSIS - Gemma Ecclesiastica. Vol. II. in BREWER, John Sherren (Ed.) - Giraldi Cambrensi. Opera. Vol. II. London: Longman, Green, Longman, and Roberts, 1862

Liber exemplorum ad usum praedicantium. Saeculo XIII compositus a quodam fratre minore anglico de provincia Hiberniae. Secundum codicem Dunelmensem editus. Ed. Andrew George Little. Aberdoniae: Typis Academicis, 1908.

PETRUS VENERABILIS - De Miraculis. in MIGNE, Jean-Paul (Ed.) - Petri Venerabilis abbatis cluniacensis noni Opera Omnia. Patrologia Latina 189. Parisiis, 1890, cols. 851-952.

Studi

BARAUT I OBIOLS, Cebrià - "Un recull de miracles de Santa Maria, procedent de Ripoll, i les Cantigues d'Alfons el Savi”. Scripta e Documenta 6 (1956), pp. 127-160.

DEXTER, Elise Forsythe - Sources of the Cantigas of Alfonso el Sabio. A thesis submitted to the Graduate School of the University of Wisconsin in partial fulfillment of the requirements for the degree of Doctor of Philosophy. Wisconsin: Madison, 1926.

DISALVO, Santiago - "Gualterus de Cluny, las Cantigas y el niño que ofrece pan a Cristo: reconsideración de una antigua colección de milagros marianos”. Hagiographica XX (2013), pp. 123-142.

FERNÁNDEZ FERNÁNDEZ, Laura - "Cantigas de Santa María: fortuna de sus manuscritos". Alcanate: Revista de Estudios Alfonsíes 6 (2008-2009), pp. 323-348.

FERREIRA, Manuel Pedro - "The Stemma of the Marian Cantigas: Philological and Musical Evidence". Bulletin of the Cantigueiros de Santa Maria 6 (1994), pp. 58-98.

FERREIRO ALAMPARTE, Jaime - "Fuentes germanicas en las 'Cantigas de Santa Maria', de Alfonso X el Sabio”. Grial 31 (1971), pp. 31-62.

FIDALGO FRANCISCO, Elvira - As Cantigas de Santa María. Vigo: Xerais, 2002.

FIDALGO FRANCISCO, Elvira - "Los animales de las Cantigas de Santa Maria. Una lectura en clave simbólica”. Revista de Literatura Medieval 29 (2017), pp. 107-127.

FIDALGO FRANCISCO, Elvira - “"Bestias e animalias de muchas maneras': animales en las Cantigas de Santa María”. in PÉREZ BARCALA, Gerardo (ed.) - 'Cui tali cura vel remedio subvenitur'. De animales y enfermedades en la Edad Media europea. Avellino: Edizioni Sinestesie, 2019, pp. 99-119. HERBERT, John Alexander - Catalogue of Romances in the Department of Manuscripts in the British Museum. London: British Museum, vol. 3, 1910.

KELLER, John E. - “A Note on King Alfonso's Use of Popular Themes in his Cantigas”. Kentucky Foreign Language Quarterly I/1 (1954), pp. 26-31. 
KITTREDGE, George Lyman - Witchcraft in Old and New England. Cambridge: Harvard University Press, 1929.

MACY, Gary - "Theology of the Eucharist in the High Middle Ages". in A Companion to the Eucharist in the Middle Ages. Leiden-Boston: Brill, 2011, pp. 365-398.

MARTINS, Mário - “O Livro dos Milagres da Bem-aventurada Virgem Maria”. Brotéria 70 (1960), pp. 517-532.

MENDES, Augusto de Carvalho - "Os Animais nas Cantigas de Santa Maria (I)”. Eikón Imago 8/2 (2015), pp. 15-166.

MONTOYA MARTÍNEZ, Jesús - "El culto a la Eucaristia y sus derivaciones mágicas en el siglo XIII". La corónica: A Journal of Medieval Hispanic Language, Literatures, and Cultures XXXVI/1 (2007), pp. 189-196.

NASCIMENTO, Augusto Aires - Milagres medievais numa colectânea mariana alcobacense. Edição crítica, tradução e estudo. Lisboa: Colibri, 2004.

NEGRI, Manuel - “Una nuova fonte per Li Miracoli de Senta Maria: il ms. Rivipullensis 193”. Critica del testo XX/1 (2017), pp. 65-103.

NEGRI, Manuel - "Rielaborazioni agiografiche alfonsine: il caso della Cantiga de Santa María 369". Carte Romanze 8/1 (2020), pp. 75-115.

PARKINSON, Stephen - “Alfonso X, Miracle Collector”. in FERNÁNDEZ FERNÁNDEZ, Laura; RUIZ SOUSA, Juan Carlos (coord.) - Alfonso X El Sabio 1221-1284. Las Cantigas de Santa María, Códice Rico, Ms. T-I-1, Real Biblioteca del Monasterio de San Lorenzo de El Escorial, El Códice Rico de las Cantigas de Santa María. Vol. II. Madrid: Editorial Testimonio, 2011, pp. 79-105.

PARKINSON, Stephen - "The Miracles Came in Two by Two: Paired Narratives in the Cantigas de Santa Maria". in CONDE, Juan-Carlos; GATLAND, Emma (ed.) - Gaude Virgo Gloriosa: Marian Miracle Literatura in the Iberian Peninsula and France in the Middle Ages. London: QMUL, 2011, pp. 65-85.

PARKINSON, Stephen - The Oxford Cantigas de Santa Maria Database [In linea]. Oxford: Centre for the Study of the Cantigas de Santa Maria, actual. 2 luglio 2020. [Consultato il 2 luglio 2020]. Disponibile all'indirizzo http://csm.mml.ox.ac.uk/?p=home.

PARKINSON, Stephen; DEIRDRE, Jackson - "Collection, Composition and Compilation in the Cantigas de Santa Maria". Portuguese Studies 22 (2006), pp. 159-172.

PONCELET, Albert - "Index miraculorum B.V. Mariae quae saec. VI-XV latine conscripta sunt". Analecta Bollandiana XXI (1902), pp. 242-360.

SCHAFFER, Martha - "Los códices de las "Cantigas de Santa María": Su problemática". in DOMÍNGUEZ RODRÍGUEZ, Ana; MONTOYA MARTÍNEZ, Jesús (eds.) - El scriptorium alfonsí: de los Libros de astrología e las 'Cantigas de Santa María'. Madrid: Editorial Complutense, 1999, pp. 127-148. THOMPSON, Stith - Motif-Index of folk-literature. A classification of narrative elements in folktales, ballads, myths, fables, mediaeval romances, exempla, fabliaux, jest-books, and local legends. Revised and enlarge edition. Bloomington: Indiana University Press, 1955-1958.

TUBACH, Frederich - Index exemplorum: a handbook of medieval religious tales. Helsinki: Academia Scientiarum Fennica, 1981. 


\section{NOTE}

1. D'ora in avanti, si utilizzerà l'acronimo CSM per riferirsi ad un singolo testo o all'opera in generale. Sul periodo di elaborazione delle CSM. FERREIRA, Manuel Pedro - "The Stemma of the Marian Cantigas: Philological and Musical Evidence". Bulletin of the Cantigueiros de Santa Maria 6 (1994), pp. 58-98; FERNÁNDEZ FERNÁNDEZ, Laura - “Cantigas de Santa María: fortuna de sus manuscritos". Alcanate: Revista de Estudios Alfonsíes 6 (2008-2009), pp. 323-348.

2. Per tutti questi aspetti: PARKINSON, Stephen - "Alfonso X, Miracle Collector". in FERNÁNDEZ FERNÁNDEZ, Laura; RUIZ SOUSA, Juan Carlos (coord.) - Alfonso X El Sabio 1221-1284. Las Cantigas de Santa María, Códice Rico, Ms. T-I-1, Real Biblioteca del Monasterio de San Lorenzo de El Escorial, El Códice Rico de las Cantigas de Santa María. Madrid: Editorial Testimonio, 2011, vol. II, pp. 79-105. Per una panoramica sulle diverse tipologie di fonti, FIDALGO FRANCISCO, Elvira - As Cantigas de Santa María. Vigo: Xerais, 2002. Su un esempio di riadattamento agiografico nelle CSM invece NEGRI, Manuel "Rielaborazioni agiografiche alfonsine: il caso della Cantiga de Santa María 369". Carte Romanze 8/1 (2020), pp. 75-115.

3. Sulle tipologie, MACY, Gary - "Theology of the Eucharist in the High Middle Ages". in A Companion to the Eucharist in the Middle Ages. Leiden-Boston: Brill, 2011, pp. 365-398.

4. Si segue, come da prassi, la numerazione dei testi nel testimone più completo dell'opera, il ms. E (Biblioteca de El Escorial, I.b.2). Sulla rimanente tradizione testuale, si rimanda a: SCHAFFER, Martha - "Los códices de las 'Cantigas de Santa María': Su problemática”. in DOMÍNGUEZ RODRÍGUEZ, Ana; MONTOYA MARTÍNEZ, Jesús (eds.) - El scriptorium alfonsí: de los Libros de astrología e las 'Cantigas de Santa María'. Madrid: Editorial Complutense, 1999, pp. 127-148. A loro volta, questi testi fanno parte di un gruppo più ampio di CSM nel quale si trovano anche prodigi in cui non avviene una trasformazione degli elementi impiegati in carne o sangue durante la celebrazione del sacramento dell'Eucaristia, ma che comunque testimoniano il potere dell'ostia per qualche guarigione o anche solo per testimoniare le verità di fede. Si tratta delle CSM 139, 222, 225, 251, 263 e 353. Sulle CSM 139 e 253, DISALVO, Santiago - "Gualterus de Cluny, las Cantigas y el niño que ofrece pan a Cristo: reconsideración de una antigua colección de milagros marianos". Hagiographica 20 (2013), pp. 123-142.

5. Non si possono dunque annoverare tra i casi di paired narratives proposti in PARKINSON, Stephen - "The Miracles Came in Two by Two: Paired Narratives in the Cantigas de Santa Maria". in CONDE, Juan-Carlos; GATLAND, Emma (ed.) - Gaude Virgo Gloriosa: Marian Miracle Literatura in the Iberian Peninsula and France in the Middle Ages. London: QMUL, 2011, pp. 65-85. Nelle CSM individuate dallo studioso inglese, infatti, a numeri diversi corrispondevano CSM del tutto simili nell'impianto narrativo, ma associate a luoghi di culto differenti. Non è il caso delle CSM 128 e 208 che, pur rifacendosi ad un medesimo panorama miracolistico, fanno capo in concreto a due diversi filoni narrativi, già autonomi a monte.

6. Per il testo, ALFONSO X, El Sabio - Cantigas de Santa María. Cantigas 101 a 260. Vol. II. Ed. Walter Mettmann. Madrid: Castalia, 1988, pp. 82-84. Per un riassunto parziale della vicenda, si vedano anche: KELLER, John E. - “A Note on King Alfonso's Use of Popular Themes in his Cantigas". Kentucky Foreign Language Quarterly I/1 (1954), pp. 29-30; MONTOYA MARTÍNEZ, Jesús - "El culto a la Eucaristia y sus derivaciones mágicas en el siglo XIII". La corónica: A Journal of Medieval Hispanic Language, Literatures, and Cultures XXXVI/1 (2007), p. 194.

7. THOMPSON, Stith - Motif-Index of folk-literature. A classification of narrative elements in folktales, ballads, myths, fables, mediaeval romances, exempla, fabliaux, jest-books, and local legends. Revised and enlarge edition. Bloomington: Indiana University Press, 1955-1958; 
TUBACH, Frederich - Index exemplorum: a handbook of medieval religious tales. Helsinki: Academia Scientiarum Fennica, 1981.

8. Per il censimento, si è partiti da: PONCELET, Albert - "Index miraculorum B.V. Mariae quae saec. VI-XV latine conscripta sunt". Analecta Bollandiana XXI (1902), pp. 249, 253, 272, 317 e 343; HERBERT, John Alexander - Catalogue of Romances in the Department of Manuscripts in the British Museum. London: British Museum, vol. 3, 1910, p. 23; DEXTER, Elise Forsythe - Sources of the Cantigas of Alfonso el Sabio. Wisconsin: Madison, 1926, p. 158. Tesi di Dottorato; KITTREDGE, George Lyman - Witchcraft in Old and New England. Cambridge: Harvard University Press, 1929, a p. 469; BARAUT I OBIOLS, Cebrià - "Un recull de miracles de Santa Maria, procedent de Ripoll, i les Cantigues d'Alfons el Savi”. Scripta e Documenta 6 (1956), p. 127; FERREIRO ALEMPARTE, Jaime - "Fuentes germanicas en las 'Cantigas de Santa Maria', de Alfonso X el Sabio”. Grial 31 (1971), pp. 52-53; PARKINSON, Stephen - The Oxford Cantigas de Santa Maria Database [In linea]. Oxford: Centre for the Study of the Cantigas de Santa Maria, actual. 2 luglio 2020. [Consultato il 2 luglio 2020]. Disponibile all'indirizzo http://csm.mml.ox.ac.uk/?p=home.

9. PETRUS VENERABILIS - De Miraculis. in MIGNE, Jean-Paul (ed.) - Petri Venerabilis abbatis cluniacensis noni Opera Omnia. Patrologia Latina 189. Parisiis, 1890, cols. 851-952, alle cols. 851-853.

10. Il miracolo poi prosegue. Dopo aver constatato che la piccola figura non dava segni di vita, l'uomo decise di dargli degna sepoltura, ma dopo averla toccata, svanì misteriosamente nel nulla. Il solo contatto con le sue mani - evidentemente considerate indegne - causò infatti la sparizione dei sacri azzimi.

11. Contiene le seguenti opere, dalla descrizione pubblicata nel sito della Bibliothèque Nationale de France, recuperabile dall'indirizzo https://archivesetmanuscrits.bnf.fr/ark:/12148/cc75683z [ultima consultazione: 26 maggio 2020]: a. Sermones; b. Versi di Thibaud de Marly sulla morte (2r); c. Exempla moralia (7v-156v); d. Sermones (114); e. Vincentii Bellovacensis expositio in orationem dominicam (122), et in Salutationem b. Marie (141v); f. [Dictamen de nomine Jesu] (153); g. Sermo decani Parisiensis factus in capitulo Sancti Victoris (169v); h. Expositio b. Gregori super Cantico (187); i. [Carmina pia] (245); 1. Versus de penitentia glosati (288); 1. Sermo magistri W. de Monte (289v).

12. Il testo è inedito. Si trascrive dalla riproduzione fotografica del codice recuperabile dal database Gallica, all'indirizzo https://gallica.bnf.fr/ark:/12148/btv1b90666494 [ultima consultazione: 26 maggio 2020]. Tra parentesi quadre si indicano le carte del codice e, con tre punti di sospensione, si segnalano i loci di difficile lettura. Le parentesi uncinate contengono invece eventuali integrazioni in corrispondenza di guasti meccanici o di mutilazioni delle carte. Gli scioglimenti delle abbreviature e dei compendi vengono invece indicati in corsiva.

13. Nell'edizione digitale della carta non sembra esserci un guasto, ma il punto non risulta del tutto leggibile per via della piega assunta dal foglio in corrispondenza della cucitura interna. Purtroppo, alla data attuale, non è stata possibile una consultazione in loco di questa sezione del codice.

14. Si integra tenendo conto di quanto scrive Pietro il Venerabile, in molti punti sovrapponibile a questa versione. Nell'edizione digitale della carta, non sembra esserci un guasto, ma il punto risulta illeggibile sempre per via della piega del codice.

15. BARAUT I OBIOLS, Cebrià - Un recull de miracles ..., p. 127. Sul concetto (e sulla pratica) della 'marianizzazione': DISALVO, Santiago - "Gualterus de Cluny, las Cantigas y el niño que ofrece pan a Cristo: reconsideración de una antigua colección de milagros marianos". Hagiographica XX (2013), pp. 123-142. 
16. Quanto a datazione e storia, si veda anche NASCIMENTO, Augusto Aires - Milagres medievais numa colectânea mariana alcobacense. Edição crítica, tradução e estudo. Lisboa: Colibri, 2004, pp. 48-56. Il secondo testimone, risalente già al sec. XIV, è proprio quello studiato da Nascimento e la cui esistenza era già stata segnalata in MARTINS, Mario - "O Livro dos Milagres da Bem-aventurada Virgem Maria". Brotéria 70 (1960), pp. 517-532.

17. La collezione che raccoglie vari miracoli mariani occupa la sesta sezione del codice pergamenaceo (la quarta, se si considera la descrizione dello stesso effettuata da Próspero de Bofarull, poi confluita nella Biblioteca Patrum Latinorum Hispaniensis). Si propone qui la descrizione più recente offerta dal Portal de Archivos Españoles (Pares) all'indirizzo http://pares.mcu.es/ ParesBusquedas20/catalogo/description/2323672: 1. [De laude psalmorum], 1rv; 2. Augustinus De Penitencia Solomonis, 2r-3r; 3. [De Pipino a Felipe II], 3r; 4. [Relación de nombres], 3v; 5. [Advocationes Virginis], 4r-27v; 6. [Miracula beatae virginis Mariae], 27v-48r; 7. Virgo pura stirps regalis, 48r; 8. [Homiliae], 48v-67v; 9. [De Officio Missae], 68r-73v; 10. [De sacramentis], 73v-81v; 11. [Sententiarum Liber I], 81v-87r; 12. [Homiliae], 87r-169v; 13. Hieronimus (...), 170r; 14. De miraculis (...) Montis Serrati, 170v-173v; 15. [Orazioni e consigli morali in latino e catalano], 173v-174v (agg. XV sec.)] (...) poi ripresa nel XIV sec. dal ms. Lisboa, Biblioteca Nacional, Alcobacense 39.

18. BARAUT I OBIOLS, Cebrià - Un recull de miracles ..., p. 127. Si riporta il testo basandosi su quello edito dallo studioso catalano. Di seguito si procede ad una trascrizione direttamente dalle carte. In questo caso, la versione trasmessa dal ms. di Ripoll non differisce sostanzialmente da quella tramandata dal già citato codice alcobacense, latore della stessa collezione e compilato, come già precisato, alcuni anni dopo.

19. Il manoscritto presenta la forma misit. Si corregge pertanto l'errore di trascrizione da parte di Baraut.

20. Baraut i Obiols inverte gli elementi del manoscritto. La sequenza che si legge è ibi stare e non stare ibi.

21. L'avverbio viene inserito nell'interlinea superiore in corrispondenza del locum tra Sacerdos e ut e viene giustamente trascritto da Baraut i Obiols a testo. Si tratta di una correzione incipiente dello stesso copista.

22. L'avverbio viene inserito nell'interlinea superiore tra locum e vas e viene giustamente trascritto da Baraut i Obiols a testo. Si tratta di una correzione incipiente dello stesso copista.

23. In questo punto, Baraut i Obiols trascrive un et, ma non sembra esserci nessuna nota tachigrafica corrispondente alla congiunzione. Pertanto, si emenda.

24. Che si dovesse ipotizzare l'esistenza di una fonte non ancora messa in relazione con tale tradizione per spiegare alcune apparenti innovazioni sul tema era già chiaro a Jaime Ferreiro Alemparte, sebbene lo studioso continuasse comunque ad ipotizzare, come base, il récit trasmesso da Cesario di Heisterbach. Le differenze presenti tra i vari récits avrebbero potuto trovare una spiegazione - sempre secondo lo studioso - nell'intrusione di almeno una terza fonte (o variante) nel processo di raccolta e composizione della CSM. FERREIRO ALEMPARTE, Jaime - Fuentes germanicas ..., pp. 52-53.

25. La 'composition' è la seconda fase del processo di elaborazione delle CSM, e riguarda, nello specifico, ciascun testo che viene elaborato e che poi, nell'ultima fase, la 'compilation', passa a formar parte dell'intera collezione mariana. Ovviamente qui si è semplificato, ma non bisogna pensare che tali processi di allestimento delle CSM siano stati così lineari. Per avere una chiara panoramica sul modo di procedere, spesso ricorsivo, dello scrittoio del Re Sapiente: PARKINSON, Stephen; DEIRDRE, Jackson - "Collection, Composition and Compilation in the Cantigas de Santa Maria”. Portuguese Studies 22 (2006), pp. 159-172.

26. Per tutti questi casi, si consiglia la consultazione di NEGRI, Manuel - "Una nuova fonte per Li Miracoli de Senta Maria: il ms. Rivipullensis 193". Critica del testo XX/1 (2017), pp. 65-103. 
27. Per una precedente lettura della CSM, si rimanda a MONTOYA MARTÍNEZ, Jesús - El culto a la Eucaristia ..., p. 193. Come viene puntualizzato in FIDALGO FRANCISCO, Elvira - "Los animales de las Cantigas de Santa Maria. Una lectura en clave simbólica”. Revista de Literatura Medieval 29 (2017), pp. 107-127 (e poi nel più recente: FIDALGO FRANCISCO, Elvira - “'Bestias e animalias de muchas maneras': animales en las Cantigas de Santa María”. in PÉREZ BARCALA, Gerardo (ed.) - 'Cui tali cura vel remedio subvenitur'. De animales y enfermedades en la Edad Media europea. Avellino: Edizioni Sinestesie, 2019, pp. 99-119), la scelta di Tolosa - luogo simbolico per il catarismo - non può che suggerire per il protagonista l'appartenenza alla comunità dei miscredenti, anticipando inoltre che quello che viene criticato attraverso questa CSM è proprio la non credenza nel dogma della transustanziazione.

28. MENDES, Augusto de Carvalho - "Os Animais nas Cantigas de Santa Maria (I)”. Eikón Imago 8/2 (2015), p. 138. Per il testo, ALFONSO X, El Sabio - Cantigas de Santa María. Cantigas 101 a 260. II. Ed. Walter Mettmann. Madrid: Castalia, 1988, pp. 257-259. Il verbo obrar (e se vos obrar sabedes, verei que faredes $i$; v. 25) - parrebbe suggerire che l'eretico fosse a conoscenza del fatto che le api, eventualmente, avrebbero iniziato a comportarsi in un certo modo al cospetto dei sacri azzimi, magari costruendovi qualcosa attorno a sua protezione (si noti l'avverbio i). L'inserimento dell'ostia nell'arnia si configurerebbe pertanto come un voler verificare, seppur in maniera errata, la sacralità del Corpo di Cristo da parte dell'eretico. Questa lettura può trovare una conferma nella dichiarazione fatta dal protagonista quando si appresta a comprovare lo stato dell'arnia una volta arrivato il momento della raccolta del miele: "Verei que obra feit' han / NA hostia as abellas"; vv. 29-30 (maiuscolo nostro). Sul verbo obrar nel contesto della lirica galegoportoghese, sia riferito ad un 'realizzare, costruire' in senso materiale, che ad un 'operare' in senso lato (dal lat. ŌPĚRĀRE). LORENZO, Ramón - La traducción gallega de la Crónica General y de la Crónica de Castilla. Orense: Instituto de Estudios Orensanos Padre Feijóo, vol. II (Glosario), 1977, p. 923.

29. GIRALDUS CAMBRENSIS - Gemma Ecclesiastica. in BREWER, John Sherren (ed.) Giraldi Cambrensi. Opera. London: Longman, Green, Longman, and Roberts, 1862, vol. II, pp. 42-43.

30. Per il testo, CAESARIUS HEISTERBACENSIS - Dialogus Miraculorum. Vol. I. Ed. Joseph Strange. Coloniae: Bonnae et Bruxellis, Sumptibus J. M. Heberle (H. Lempertz \& Comp.), 1851, pp. 172-173.

31. Per una breve sinossi della vicenda, FERREIRO ALEMPARTE, Jaime - Fuentes germanicas ..., p. 52.

32. A titolo di esempio, si riportano qui solamente i passaggi fondamentali del prodigio così come trasmesso dal Liber exemplorum e facenti capo al momento dell'immissione dell'ostia in un albero, del suo ritrovamento e della constatazione della presenza di una struttura votiva attorno ad essa: "Nostris quoque diebus in Alamannie partibus accidit quendam eukaristiam sibi datam vel fidei defectu vel diffidencia in arbore quadam orti sui concava reposuisse (...) invenit tanquam in medio operis congesti ecclesiam ceream, nulla tamen omnino mellis gutta perfussam, turrem habentem et tintinnabula, altare quoque et calicem desuper, cum corporalibus et patena, tantum quoque thesaurum minus ante discrete reconditum quam pro loci natura honestius poterat, non solum apicularum officio set etiam angelorum ministerio, Deo auctore, in calicem repositum (...)". Liber exemplorum ad usum praedicantium. Saeculo XIII compositus a quodam fratre minore anglico de provincia Hiberniae. Secundum codicem Dunelmensem editus. Ed. Andrew George Little. Aberdoniae: Typis Academicis, 1908, p. 9.

33. ÉTIENNE DE BOURBON - Tractatus de diversis materiis praedicabilibus. Ed. Albert Lecoy de la Marche. Paris: Librairie Renouard, 1877, pp. 266-267.

34. FERREIRO ALEMPARTE, Jaime - Fuentes germânicas ..., pp. 52-53. 
35. Come è ovvio, si tratta solamente di una ipotesi suggerita anche da quello che si riporta a continuazione. Nulla vieta comunque di prendere in considerazione l'esistenza di fonti parallele o di 'cugini narrativi' che avrebbero potuto favorire questo processo di diversificazione. Le ricerche di questo tipo sulle CSM sono sempre aperte a nuove suggestioni ed ipotesi che vanno aumentando o perfezionandosi anche al crescere del volume di testi e di miracoli mariani (ma non solo) censiti dalla critica.

36. HERBERT, John Alexander - Catalogue of Romances ..., p. 648.

37. MENDES, Augusto de Carvalho - "Os Animais nas Cantigas de Santa Maria (I)"..., p. 136.

38. Si rimanda sempre alle tabelle riassuntive presentate nelle pagine anteriori.

\section{RIASSUNTI}

Le Cantigas de Santa María 104, 128, 147 e 208 presentano dei miracoli eucaristici dove delle ostie subiscono una trasfigurazione. Le loro fonti non sono ancora state ben definite dalla critica. Questo studio vuole offrire delle nuove ipotesi sui precedenti delle Cantigas 128 e 208, incentrate sul motivo dell'adorazione di un'ostia consacrata da parte di alcune api. Se per la Cantiga 128 si evidenzia l'influenza di una versione contenuta in una collezione miracolistica di provenienza catalana, in quanto portatrice di dettagli condivisi in esclusiva col testo galego medievale, per la Cantiga 208 viene ribadita la presenza, alla base, dell'opera del cistercense tedesco Cesario di Heisterbach, con alcune innovazioni da imputare forse alla stessa corte del sovrano per far fronte ad alcune esigenze dottrinali del tempo.

The Cantigas de Santa María 104, 128, 147, and 208 present Eucharistic miracles where the hosts undergo a transfiguration. Their sources have not yet been well defined by scholars. This study aims to offer new hypotheses on the precedents of Cantigas 128 and 208, centered on the motif of the adoration of a consecrated host by some bees. If for Cantiga 128 the influence of a version contained in a miraculous collection of Catalan origin is highlighted, as it bears details shared exclusively with the medieval Galician text, for Cantiga 208 the presence is reiterated, at the base, of the work of the Cistercian monk Cesario of Heisterbach, with some innovations to be attributed to the same court of the sovereign to perhaps some doctrinal needs of his time.

\section{INDICE}

Keywords : Cantigas de Santa María, Alfonso X, Eucharistic miracles, Dialogus miraculorum, Rivipullensis 193

Temi : Cantigas de Santa María, Alfonso X, Miracoli eucaristici, Dialogus miraculorum, Rivipullensis 193 


\section{AUTORE}

\section{MANUEL NEGRI}

Universidade de Santiago de Compostela, Facultade de Filoloxía, Departamento de Filoloxía Galega C.P. 15701, Santiago de Compostela, España. m.negri@usc.es. https://orcid.org/ 0000-0003-3532-9810 